\title{
La pertinence de tenir compte du sexe des " opérateurs » dans les études ergonomiques: Bilan de recherches
}

The relevance of gender in ergonomic studies: A summary of research results La pertinencia de tomar en cuenta el sexo de los « operadores » en los estudios ergonómicos : balance de investigaciones

\section{Karen Messing}

\section{OpenEdition}

1 Journals

Édition électronique

URL : http://journals.openedition.org/pistes/3840

DOI : $10.4000 /$ pistes.3840

ISSN : 1481-9384

Éditeur

Les Amis de PISTES

Édition imprimée

Date de publication : 1 novembre 1999

\section{Référence électronique}

Karen Messing, "La pertinence de tenir compte du sexe des « opérateurs » dans les études

ergonomiques: Bilan de recherches », Perspectives interdisciplinaires sur le travail et la santé [En ligne],

1-1 | 1999, mis en ligne le 01 novembre 1999, consulté le 20 avril 2019. URL : http://

journals.openedition.org/pistes/3840; DOI : 10.4000/pistes.3840

Ce document a été généré automatiquement le 20 avril 2019.

\section{cc)}

Pistes est mis à disposition selon les termes de la licence Creative Commons Attribution - Pas d'Utilisation Commerciale - Pas de Modification 4.0 International. 


\title{
La pertinence de tenir compte du sexe des « opérateurs » dans les études ergonomiques : Bilan de recherches
}

\author{
The relevance of gender in ergonomic studies: A summary of research results \\ La pertinencia de tomar en cuenta el sexo de los « operadores » en los estudios \\ ergonómicos : balance de investigaciones
}

Karen Messing

\section{NOTE DE L'AUTEUR}

Une version préliminaire de ce texte a été publiée dans Messing (1996).

J'ai profité de discussions avec plusieurs ergonomes, notamment Nicole Vézina, Ana Maria Seifert et Ghislaine Doniol-Shaw. J'ai été influencée par des sociologues, dont Danièle Kergoat, Lucie Dumais et Angelo Soares. Notre partenariat, "l'Invisible qui fait mal » comprend Gisèle Bourret et Nicole Lepage de la CEQ, Marie-France Benoit et Jocelyne Everell de la CSN, Carole Gingras et JeanPierre Néron de la FTQ, ainsi que les chercheures universitaires Diane Demers, Nancy Guberman, Katherine Lippel, Donna Mergler, Karen Messing, et Nicole Vézina. Il est coordonné par Sylvie de Grosbois des Services aux collectivités de l'Université du Québec à Montréal. Le partenariat est subventionné par le Conseil québécois de recherche sociale. Plusieurs recherches évoquées ici ont été subventionnées par l'Institut de recherche en santé et en sécurité du travail, le Conseil de recherches en sciences humaines, et le Fonds FCAR. 


\section{Introduction}

1 L'analyse ergonomique est probablement l'outil le plus puissant utilisé actuellement pour intervenir en santé au travail dans le monde francophone. De popularité croissante, elle a été employée dans beaucoup de milieux pour «comprendre le travail pour le transformer " (Guérin et coll., 1991). Les formations données et les recherches effectuées par des ergonomes, notamment au Conservatoire national des arts et métiers, ont sûrement eu un effet très positif sur la santé des femmes et des hommes au travail. Dès le début, plusieurs travaux ont permis d'identifier des exigences et contraintes du travail des femmes. Par exemple, on a étudié les travailleuses à la chaîne (Wisner et coll., 1967), les couturières (Teiger et Plaisantin, 1984), les téléphonistes (Dessors et coll., 1978), les préposées à la saisie de données (Teiger et Bernier, 1992) et le travail domestique des femmes (Doniol-Shaw, 1983). Mais, en général, les ergonomes n'ont pas prêté une attention particulière au sexe des "opérateurs ». La population des opérateurs étant diverse de plusieurs points de vue, le sexe est considéré parmi un ensemble de caractéristiques. Classer la population selon le sexe paraîtrait comme une façon réductionniste de traiter de la diversité que l'on retrouve chez les populations au travail. Par contre, les ergonomes se sont impliqués dans des travaux qui considèrent la population ouvrière selon l'âge (Derrienic et coll., 1996).

2 En sociologie, la division sexuelle du travail a été souvent traitée sous l'angle de l'accès à l'égalité, que ce soit au Québec (David, 1986; Vandelac, 1985) ou en France (travaux de Kergoat [1983] et du Groupe d'étude de la division sociale et sexuelle du travail du CNRS). On voit que les femmes sont concentrées dans le secteur des services, et qu'elles se retrouvent, le plus souvent, dans les emplois de bureau et les services personnels. Elles sont sous-représentées au niveau des cadres et professions intellectuelles supérieures (Asselin et coll., 1994 ; Armstrong et Armstrong, 1993 ; Saurel-Cubizolles et coll., 1996). Elles font toujours beaucoup plus de travail domestique, la différence étant moins prononcée au Québec (Le Bourdais et coll., 1987; Tissot et coll., 1997) qu'en France (Derrienic et coll., 1996, p. 380).

3 En même temps, des sociologues québécoises (Guyon, 1997) et françaises (SaurelCubizolles et Blondel, 1996) démontrent les différences entre les hommes et les femmes du point de vue de la santé. À titre d'exemples, les hommes sont atteints plus souvent de crises cardiaques, d'accidents et de certains cancers alors que les femmes ont plus souvent souffert d'accidents cardiovasculaires, de problèmes musculo-squelettiques, de problèmes psychologiques et de certains autres cancers. Ces différences ont parfois été associées à des conditions de travail rémunérées et non-rémunérées (de Koninck, 1983 ; Saurel-Cubizolles et coll., 1996).

4 Parce que les hommes et les femmes sont distribués de façon différentielle dans les milieux de travail et qu'ils montrent des effets différents de leur travail, quelques ergonomes commencent à poser la question de la place du sexe dans les études ergonomiques (Messing, 1996 ; 1999 ; Kilbom et coll., 1998 ; Gonik et coll., 1998). Depuis 1989, notre groupe de recherche, le CINBIOSE, effectue des recherches ergonomiques sur la division sexuelle du travail et sur le travail des femmes. Nous avons trouvé que les postes et les tâches varient selon le sexe, qui a aussi une pertinence pour comprendre les modes opératoires. Nous concluons que, non seulement la considération du sexe des "opérateurs" ne les réduit pas en catégories, elle fournit au contraire une façon 
d'enrichir l'analyse ergonomique. Les exemples utilisés pour démontrer ces thèses proviennent des recherches effectuées au CINBIOSE dans le cadre d'un programme de recherche, «l'Invisible qui fait mal», mené en collaboration avec les trois principales centrales syndicales du Québec. Nous avons considéré les femmes qui cherchent à intégrer les postes anciennement masculins et les femmes dans les métiers traditionnellement féminins.

\section{Les femmes dans les métiers anciennement masculins}

5 Nos recherches ergonomiques sur la division sexuelle des tâches explorent: (1) La possibilité d'aménager des postes traditionnellement masculins pour que les femmes puissent y avoir accès ; (2) La place du sexe dans la répartition du travail collectif.

\section{Les femmes dans les postes « masculins " de cols bleus}

6 La possibilité que des femmes prennent des postes traditionnellement masculins pose la question des différences hommes-femmes. La sociologue Dominique Gaucher a énuméré les «qualités naturelles » attribuées aux femmes pour justifier la division sexuelle du travail (Gaucher, 1983). On engagerait des femmes quand il s'agit de soins aux personnes, de travail à l'intérieur, de travail perçu comme ne présentant aucun danger, des postes immobiles, de travail qui exige de l'acuité visuelle, de la dextérité, de la vitesse d'exécution, de la tolérance face aux interruptions et de la capacité de faire plusieurs choses à la fois. D'autres études ont ajouté la capacité de calmer ou d'amadouer des clients agressifs (Soares, 1995) et la tendance à mettre les besoins des autres avant les siens (Carpentier-Roy, 1991).

7 S'agit-il de préjugés non-fondés, d'exigences spécifiques faites aux femmes, de résultats d'une éducation sexualisée, ou de modes opératoires différents selon le sexe ? La capacité inférieure de la plupart des femmes pour lever des charges importantes serait-elle attribuable au manque d'exercice ou aux hormones? L'éducation des deux sexes diffère trop chez l'humain pour qu'on puisse distinguer les différences innées. Déficeler les apports du sexe biologique et de l'éducation aux comportements en société serait une tâche compliquée et ingrate, puisque l'origine de ces différences n'a aucune importance pour les travailleurs et travailleuses. Ce qui leur importe, c'est la prise en charge des différences hommes/femmes dans le milieu de travail.

Or, on peut constater que le milieu de travail ne s'adapte pas toujours aux spécificités biologiques des femmes. Malgré le fait que la majorité des femmes enceintes travaillent, les postes de travail ne sont pas habituellement conçus de façon à répondre aux exigences de leur état (Paul, 1993). Au Québec, une loi prévoit la réaffectation, le retrait ou le réaménagement de poste des travailleuses enceintes exposées à un danger pour ellesmêmes ou pour leur fotus. Le fait qu'un tiers des femmes enceintes se prévaut de cette loi, la plupart invoquant des conditions « ergonomiques » démontre une incompatibilité entre plusieurs postes (environ $20 \%$ ) et la grossesse (Malenfant, 1996). Il est particulièrement frappant que, dans plusieurs milieux de travail, la loi soit invoquée à chaque grossesse, sans que les postes soient aménagés. En effet, au moins quatre fois sur 
cinq, la femme est retirée du travail et le poste reste inchangé (Lippel et coll., 1996). Bien que nous puissions comprendre que de petits employeurs n'adaptent pas leurs postes pour l'ensemble de l'éventail des capacités humaines, nous pensons que les grandes entreprises qui emploient beaucoup de femmes (secrétaires, vendeuses, caissières, par exemple) pourraient adapter leurs postes.

D'autres différences biologiques ne sont pas aussi absolues mais, selon nos recherches, elles conditionnent l'interaction avec le poste de travail. La taille par exemple peut déterminer la possibilité d'effectuer certaines tâches sans douleur (Courville et coll., 1991 ; 1992 ; Chatigny et coll., 1995). Nous avons étudié le travail des cols bleus dans une municipalité où quelques femmes sont entrées depuis peu de temps (Messing et coll., 1994a ; Dumais et Courville, 1995). D'un échantillon de 113 personnes, toutes les femmes étaient plus petites que la moyenne des hommes et tous les hommes étaient plus grands que la moyenne des femmes. Bien que l'écart de grandeur à l'intérieur d'un sexe ait été supérieur à la différence de moyennes entre les deux sexes, on pouvait prévoir des problèmes lors de l'intégration des femmes dans des métiers traditionnellement masculins, où les outils, les aménagements et les équipements avaient été conçus en fonction du gabarit masculin.

10 Nous avons pu apparier des hommes et des femmes engagés à une même fonction, la plupart comme jardiniers, journaliers ou nettoyeurs. Parmi les 60 paires identifiées, une minorité appartenait toujours à la catégorie d'emploi d'origine, quelques années après l'embauche. Au moment de l'entrevue, les hommes surtout s'étaient répartis dans un grand nombre de métiers. Pour les 34 paires qui étaient demeurées au même emploi, il y avait eu divergence au niveau des tâches. Les jardiniers rapportaient qu'ils plantaient des arbres, tondaient le gazon, opéraient de la machinerie et taillaient des arbres, alors que les jardinières plantaient des petites plantes, désherbaient des plates-bandes et taillaient des arbustes. Selon les entrevues des 113 personnes, il y a eu une redistribution des tâches suite à l'introduction des femmes, dans $50 \%$ des équipes de travail. Les femmes éprouvaient des difficultés à avoir accès à certaines conditions qui facilitent le travail, à cause d'objections à leur présence. Selon une analyse des accidents de travail et des entrevues, la distribution différentielle des tâches avait des conséquences pour les accidents et les problèmes musculo-squelettiques (Messing et coll., 1994b). Et, comme les hommes les plus petits, des femmes cols bleus se trouvaient désavantagées par des outils trop grands pour leur main, des bottes trop grosses, un volant trop loin du siège d'un chariot, etc.

Boucher (1995) a poursuivi l'étude en étudiant les modes opératoires d'une équipe de jardiniers et de jardinières de gabarits physiques différents. Elle a examiné en plus grand détail les modes opératoires d'une équipe de quatre personnes, dont un homme et une femme ayant le gabarit typique de leur sexe, un homme de taille plus grande que la moyenne des hommes mais de poids plus léger $(182 \mathrm{~cm}, 56 \mathrm{~kg})$ et une femme de taille plus petite que la moyenne des femmes mais de poids plus élevé $(155 \mathrm{~cm},>75 \mathrm{~kg})$. Elle a trouvé que les modes de préhension des outils et les postures de travail étaient déterminés par bien d'autres facteurs que le sexe. Par exemple, la jardinière qui pesait plus que les trois autres pouvait utiliser son poids en complément à la force de ses bras, pour enfoncer la bêche. Les modes de préhension de la bêche représentaient un compromis entre les conditions d'exécution du travail et l'état de la personne. La posture de travail représentait un compromis entre la nécessité d'exercer une force adéquate, l'état de fatigue, le confort postural et les contraintes des situations rencontrées (outils et 
aménagements des parcs), mais aussi entre les tâches assignées par le contremaître et la situation de la personne dans l'équipe (compétences, ancienneté, sexe). La répartition des tâches dans les équipes se faisait selon un certain nombre de critères ayant trait, non seulement aux caractéristiques physiques, mais aussi aux stéréotypes.

Nous avons conclu à la nécessité d'adapter les outils et les aménagements, afin que l'ensemble des jardiniers et des jardinières puissent travailler en équipe de façon égale et sans douleur (Boucher et coll., 1994). Cependant, il n'y avait pas toujours la volonté de procéder aux adaptations. Un superviseur, par exemple, nous a dit, sans retenue, que les femmes ne pouvaient pas être émondeurs parce que l'équipement n'était pas adapté à leur taille et qu'il n'avait aucune intention de demander l'équipement nécessaire.

Notons que l'inadéquation des postes avec le corps des femmes n'est pas l'apanage exclusif des postes non-traditionnel. Même dans les postes majoritairement féminins, on peut avoir l'impression que le poste a été conçu pour des personnes plus grandes que la femme moyenne. Par exemple, la majorité des caissières de banque rapportent que leur comptoir est trop large, ce qui amène des douleurs aux membres supérieurs. Plus les caissières sont petites, moins elles sont satisfaites de l'aménagement de leur poste (Seifert et coll., 1996a). De même, une étude de la transformation de la volaille démontre que les femmes sont significativement plus nombreuses que les hommes à trouver que leur poste n'est pas adapté à leur taille (Saurel-Cubizolles et coll., 1991).

En conclusion, nous avons trouvé que le sexe biologique, et ce qu'il comporte en termes de caractéristiques anthropométriques est un déterminant parmi d'autres de l'activité de travail dans un métier nouvellement intégré par les femmes. Par contre, les représentations des capacités des deux sexes, et parfois les stéréotypes, sont très importants en ce qui concernent la répartition du travail dans le collectif ainsi que la disponibilité des équipements appropriés.

\section{Les femmes dans les postes « masculins »: les aspects collectifs}

Nous avons poursuivi nos recherches dans une profession où l'intégration des femmes a été faite surtout dans la période 1982-95 (Messing et Elabidi, 1998). Autrefois, les préposés aux bénéficiaires des hôpitaux étaient affectés aux « travaux lourds » qui impliquaient la manutention des bénéficiaires tandis que les préposées étaient affectées aux « travaux légers » de soins personnels. Après la fusion, Lortie (1987) a constaté que les préposées et les préposés avaient le même taux d'accidents mais n'avaient pas le même site d'accidents (haut du dos pour les femmes, bas du dos pour les hommes). Elle a expliqué ces résultats par une différence dans les tâches et les pratiques de manutention entre les deux sexes.

Plus récemment, Cloutier et Duguay (1996) ont comparé les accidents survenus en 1987 et 1991 chez le personnel des établissements de santé. Les préposées aux bénéficiaires ont davantage d'accidents de travail que les préposés, et l'écart s'élargit depuis 1987, avec une augmentation du taux pour les femmes et une diminution pour les hommes. Les accidents arrivent surtout $(63 \%)$ au dos chez les jeunes, hommes et femmes, provenant d'efforts excessifs en manipulant une personne. Contrairement à l'étude de Lortie, les statistiques disponibles ne distinguent pas entre le bas et le haut du dos, et nous ne pouvons pas savoir s'il s'agit du même type d'accidents pour les deux sexes. 
17 Lors de sessions de formation syndicales en santé et en sécurité du travail, plusieurs préposés ont dit que les préposées ne faisaient pas leur part, exposant les hommes à un surcroît de tâches. Certaines femmes disaient avoir de la difficulté à effectuer les nouvelles composantes lourdes de leur tâche. La fusion serait donc un danger pour la santé des deux sexes. Nous avons donc entrepris d'observer le travail des préposés-es aux bénéficiaires pendant 61 heures dans deux établissements, afin de documenter la distribution des tâches selon le sexe et les échanges de services entre les membres des équipes.

Nous avons constaté un très grand niveau de partage de tâches et d'informations parmi les membres du personnel de soins. Sur 1169 opérations, 523 (47 \%) étaient partagés. La collaboration dans la catégorie trouvée la plus difficile par les préposés-es (transferts, repositionnements et remontées des patients) atteint $80 \%$ des opérations. Mais plusieurs opérations difficiles s'effectuent seuls(es), sans aide mécanique, avec un risque concomitant de blessures. Les déterminants du travail difficile effectué seul étaient multiples: pression du temps, aide non disponible au moment voulu, relations hiérarchiques qui empêchent de demander de l'aide des infirmières, manque d'informations concernant le patient, manque d'équipements de manutention ou ignorance de leur fonctionnement, politiques et philosophie de l'établissement (trois fois plus de manutentions seules dans l'un des deux hôpitaux), préférences des préposés-es.

Nous avons ensuite examiné l'interaction entre le sexe et le degré de collaboration. Si l'on compare le travail effectué par les hommes et les femmes pendant la période la plus occupée du quart de jour, on voit de légères différences dans le degré de collaboration. Contrairement à ce que nous avions entendu, les femmes font un peu plus que leur part, même dans les opérations difficiles (tableau 1).

Tableau 1. Le partage hommes/femmes entre $7: 30-10: 30$

\begin{tabular}{|l|l|l|}
\hline & Préposées & Préposés \\
\hline Toutes opérations & 186 en $11.7 \mathrm{~h}$ & 187 en $14.8 \mathrm{~h}$ \\
\hline Toutes opérations effectuées seules & $117(63 \%)$ & $103(55 \%)$ \\
\hline Opérations difficiles & 42 & 48 \\
\hline Opérations difficiles effectuées seules & $16(38 \%)$ & $18(38 \%)$ \\
\hline Opérations/h & 15.9 & 12.6 \\
\hline Opérations difficiles effectuées seules/h & 1.4 & 1.2 \\
\hline
\end{tabular}

20 Contrairement aussi à la perception générale, les infirmières ne faisaient pas systématiquement appel aux hommes plus qu'aux femmes, même pour les opérations difficiles ; au contraire, il y avait une différence statistiquement significative dans l'autre sens.

21 Lors de la restitution des résultats, nous avons essayé de trouver des explications de la différence entre nos chiffres et la perception du milieu. Parmi ces explications, nous 
retrouvons celle des stéréotypes. Certaines personnes n'ont pas inclus les femmes qui faisaient beaucoup d'opérations exigeantes dans la catégorie « femmes ».

Nous avons rencontré le personnel d'une unité où l'on affirmait que les femmes ne faisaient pas leur part. Le groupe, composé de 3 hommes et 2 femmes, a porté jugement sur 8 personnes de leur unité quant à leur capacité de collaborer à des tâches physiques. Ils ont limité l'exercice à des préposés aux bénéficiaires (PAB) qu'ils connaissaient assez bien pour juger de leur capacité de travail. Trois hommes et deux femmes étaient considérés comme d'excellents collaborateurs, tandis qu'une femme, "Rosa», et un homme, "Nicolas ", étaient considérés comme incapables de faire leur part et une autre femme était considérée comme capable de faire sa part mais indésirable à cause de son mauvais caractère. Donc le fait que la même proportion des hommes et des femmes de leur entourage étaient considérés comme physiquement capables de faire leur part n'a pas empêché les hommes et les femmes de ce groupe de considérer que les femmes ne font pas leur part pour des raisons d'incapacité physique. Ce qui peut avoir joué dans ce cas, c'est le fait que « Rosa », âgée de près de 50 ans, insiste sur son incapacité de faire sa part, qu'elle explique par son sexe et son âge.

Une deuxième explication s'applique à certaines unités, où il a été suggéré que les hommes ont des cas plus difficiles que les femmes.

"C'est automatique, le plus gros de l'équipe a les cas lourds. »

Nous n'avons pas pu considérer l'ensemble des caractéristiques des patients dans nos statistiques.

Une troisième explication, à notre avis la plus vraisemblable, est que, les hommes sont effectivement assignés à des tâches à exigences physiques extrêmes, mais que ces extrêmes sont trop rares pour apparaître dans nos statistiques. Nous avons été témoin de deux exemples possibles de ce type de préférence pour un homme. Un soir, dans un département de patients relativement autonomes, il y avait quatre préposés, un homme et 3 femmes, avec 3 infirmières. L'assistante infirmière-chef effectuait une prise de sang auprès d'une patiente qui est devenue violente. Elle l'a attachée d'un côté et a fait appel au préposé masculin, « Roger », un homme assez costaud dans la trentaine. Une préposée a entendu des cris et est venue aider en tenant la patiente de l'autre côté. Mais c'est à l'homme qu'il revenait de tenir le bras immobile pour la piqûre. De même, nous avons observé que deux préposées ont fait appel au préposé masculin dans une situation d'urgence où une patiente était en train de tomber, et ceci, malgré la présence d'une troisième préposée et malgré le temps requis pour chercher le préposé.

Ces exemples démontrent que, dans des situations extrêmes, il se peut fort bien que l'on fasse appel préférentiellement aux hommes. Les préposés masculins ont pu nous fournir d'autres exemples de ce type de situation. Nous pouvons supposer que, dans des situations qui exigent un degré exceptionnel de force ou de courage, les hommes sont préférentiellement sollicités. Ces situations seraient peu nombreuses, donc peu présentes dans nos relevés, mais elles marqueraient la perception des préposés-es. Il est probable que ces mêmes situations posent un risque exceptionnel de blessure. Par contre, peutêtre parce que les femmes se font reprocher de ne pas "faire leur part ", elles auraient tendance à faire plus d'opérations à l'heure que les hommes, courant le risque d'usure prématurée que nous retrouvons dans l'étude de Cloutier et Duguay (1996).

Nous avons donc conclu, à partir de l'observation et de l'analyse du travail dans des équipes mixtes, que la représentation stéréotypée des capacités joue dans la répartition 
des tâches à composante physique, au-delà des différences physiologiques réelles. Pour diminuer les exigences physiques des tâches des préposés-es, nous avons proposé plusieurs pistes de solutions "classiques»: des changements au niveau du contexte d'utilisation d'aides mécaniques, des affectations de personnel, de l'étalement des opérations dans le temps, de l'élimination de certaines opérations exigeantes. Nous recommandons aussi de favoriser explicitement le travail en équipe, par la stabilisation des horaires de travail et l'aménagement de l'organisation du travail. À ces recommandations nous avons joint une suggestion de discuter des stéréotypes, que nous avons pu mettre en œuvre dans certaines sessions de formation syndicales (Confédération des syndicats nationaux - CSN). Soulignons cependant que, lors des recherches et des sessions de formation, nous avons trouvé difficile de mener ces discussions de la contribution relative des deux sexes en groupes mixtes. Nous pensons qu'il faut considérer la pertinence de faire une partie de la restitution en groupes homogènes par rapport au sexe.

\section{Les femmes dans les métiers « féminins »}

Nos recherches ergonomiques explorent aussi les exigences physiques, mentales et émotionnelles $\mathrm{du}$ travail traditionnellement féminin. Nous voulons fournir un complément nécessaire aux travaux traditionnels en ergonomie, qui touchent les exigences retrouvées souvent dans les emplois des hommes: force physique exercée de façon ponctuelle, surveillance des machines, décisions visiblement techniques, etc. Nous avons donc examiné le travail répétitif, la gestion simultanée de plusieurs contraintes, la conciliation des responsabilités professionnelles et domestiques ainsi que le travail émotionnel.

\section{Travail physique et emplois des femmes}

Notre réflexion sur les exigences physiques du travail habituellement assigné aux femmes a commencé avec une étude dirigée par Nicole Vézina, effectuée à la demande d'une association paritaire de l'habillement, suite au constat de plusieurs troubles musculosquelettiques chez des couturières (Vézina et coll., 1997). La fréquence élevée de lésions musculo-squelettiques à l'épaule gauche chez celles qui occupent les postes de couture interne et externe des jambes de pantalons a permis de cibler ces postes. Des observations nous ont permis de décrire très précisément les différentes opérations des cycles de travail, de comparer l'ordre de ces opérations d'une opératrice à l'autre, et d'illustrer la posture de travail ainsi que les mouvements exécutés par les travailleuses au cours de chacune des opérations. Les efforts dynamiques ont été comptabilisés en faisant la sommation de poids de tout ce qui était soulevé, poussé ou tiré en mesurant la force minimale requise pour déplacer les objets à l'aide d'un dynamomètre. Par exemple, la force totale exercée par les membres inférieurs au cours d'une journée a été calculée en multipliant le nombre de jambes de pantalons cousus par la fréquence d'activation de la pédale lors de la couture d'une jambe de pantalon et par la force minimale requise pour activer la pédale. Notons que ces mesures sous-estiment la force totale exercée par les couturières puisque nous n'avons pas pu considérer le temps de maintien de la force. De plus, certains efforts n'ont pu être mesurés à cause des limites d'utilisation des dynamomètres. 
30 Par cette méthode, nous avons démontré qu'une opératrice de machine à coudre peut répéter le même cycle de travail plus de 1500 fois par jour et soulever plus de 400 kilos de matériel. Quand on considère l'ensemble des efforts, elle exerce une force de plus de 2850 kilos avec ses bras, ses épaules et ses mains. Elle exerce également une force de $29648 \mathrm{~kg}$ (poste de couture externe) par les membres inférieurs pour l'activation de la pédale.

31 Si on considère le travail musculaire d'un point de vue énergétique, on le classe facilement comme léger, les objets soulevés ayant un faible poids unitaire. Par contre si l'on considère l'importance du cumul de poids soulevé et de l'effort déployé par les membres supérieurs ainsi que l'importance du nombre de mouvements répétés par les membres supérieurs, souvent dans des postures à risque (abduction, adduction), on constate bien l'importance des contraintes et de la charge de travail. Si le travail paraissait léger quand on ne considère que la dépense énergétique, il s'avérait dorénavant lourd en ce qui concerne la sollicitation ostéoarticulaire, celle de l'épaule gauche en particulier. Ce type de travail demande peut-être moins de force physique ponctuelle que le travail exécuté par les hommes (manœuvres) mais l'importance du travail accompli par les couturières dans des conditions difficiles laisse supposer une grande résistance et endurance physique.

D'autres études, effectuées chez des travailleurs et travailleuses de la transformation de la viande (Vézina et coll., 1995 ; Messing et coll., 1998a) dans une biscuiterie (Dumais et coll., 1993), et du nettoyage (Messing et coll., 1993; 1998b), ont permis de confirmer notre impression d'une division sexuelle des exigences physiques. On peut généraliser en disant que les femmes se retrouvent plus souvent là où les mouvements sont rapides, fins et sans micro-pause, et les hommes, là où il faut déployer un effort plus accentué mais plus ponctuel.

Dans la transformation de la volaille, les postes des hommes seraient aussi plus «limitants» et ceux des femmes, plus "usants» (Vézina et coll., 1995). Davantage d'accidents sont déclarés aux postes des hommes puisque leurs exigences

« peuvent représenter une barrière, une limite qui entraîne l'arrêt du travail ».

Par contre, on retrouve plus de douleur aux postes des femmes, parce que

« elles peuvent continuer à exécuter le travail malgré l'inconfort ressenti ».

Les femmes continueraient à user leur système musculo-squelettique jusqu'à ce qu'elles ne puissent plus du tout faire ce type de travail.

36 Il est difficile d'imaginer des standards pour ce type d'exigence, analogues à ce qui existent pour la levée des charges. Doit-on considérer le nombre de répétitions identiques ou la sollicitation des mêmes structures? Comment considérer les pauses et les micropauses? Quelle importance accorder au poids des objets légers?

37 Le travail statique est une autre composante des emplois féminins qui est difficile à cerner. Par exemple, le travail debout statique est caractéristique des caissières de supermarché, des caissières de banque et des vendeuses en Amérique du nord. Chez les caissières de banque, cette posture a été associée à un niveau élevé de douleurs au dos, aux jambes et aux pieds (Seifert et coll., 1997). Mais la travailleuse n'est pas complètement immobile, se déplaçant de quelques pas seulement à la fois, ce qui rend difficile la description du travail et sa transformation (Vézina et coll., 1994; Laberge et Vézina, 1998 ; Messing et Kilbom, soumis).

38 L'invisibilité relative des contraintes physiques des postes de femmes pose un problème spécifique pour la reconnaissance des problèmes et pour la recherche de solutions. Cette 
reconnaissance s'avère difficile au niveau de la prévention (Messing et Boutin, 1997) de la compensation (Lippel et coll., sous presse) et aussi dans le milieu de travail. Par exemple, une étude suédoise a démontré que la charge physique du travail des hommes s'allège avec le temps, contrairement à celle des travailleuses (Kilbom et Torgén, 1996). Effectivement, dans plusieurs milieux de travail, il existe des postes consacrés formellement ou informellement pour les hommes âgés ou accidentés, alors que la même facilité n'est pas accessible aux femmes (Messing et coll., 1993). Les exigences du travail des femmes paraissent banales et un problème au niveau du travail des femmes peut être facilement assimilé à leur sexe. Dans ces postes, les ergonomes doivent d'abord faire reconnaître les exigences du travail avant de pouvoir procéder à sa transformation.

\section{Travail mental et emplois des femmes}

Notre partenariat «L'invisible qui fait mal » a aussi examiné les contraintes mentales du travail des femmes. Après analyse du travail des enseignantes de niveau primaire, de caissières de banque, des réceptionnistes et des téléphonistes, nous avons retrouvé un certain nombre de contraintes que nous pensons caractéristiques de plusieurs emplois majoritairement féminins : être assignée à des tâches qui se chevauchent dans le temps, subir des interruptions fréquentes, avoir à répondre à plusieurs personnes à la fois, subir des conditions qui rendent très difficile la conciliation travail-famille, être un tampon entre une institution et les clients, subir de l'agression verbale de la part des clients (Messing et coll., 1997 ; Seifert et coll., 1997 ; Seifert et coll., 1996b ; Prévost et coll., 1997).

Nous avons étudié, avec la Centrale des enseignants et enseignantes du Québec (CEQ), le travail des enseignantes de niveau primaire, un emploi réputé facile et peu exigeant (Messing et coll., 1997). Nous y avons trouvé un cumul d'exigences, mais dont aucune ne parait dramatique. Les enseignantes doivent à la fois s'occuper de l'enseignement, de la discipline, du confort physique et du soutien affectif de leurs élèves. Le rythme de travail est très élevé, allant d'une moyenne de $8 \mathrm{sec}$ par intervention en $1^{\text {ère }}$ année à $14 \mathrm{sec}$ en $6^{\text {e }}$. Elles doivent faire preuve d'une concentration à toute épreuve dans une ambiance physique souvent inconfortable et bruyante. Elles doivent adapter la matière à des élèves de langues, de comportements et d'aptitudes fort différents. Elles travaillent debout sans relâche, pliant le tronc à des degrés différents selon la taille des élèves. Ce cumul, tout en ne comprenant aucune exigence impressionnante, a comme résultat un niveau élevé de détresse psychologique. Cependant, en l'absence d'analyse du travail, cette détresse est difficilement explicable puisqu'elle n'est pas associée à un déterminant précis.

\section{Travail mental et conciliation travail-famille}

Un autre aspect invisible du travail des femmes relève de la conciliation des responsabilités professionnelles et familiales, habituellement exclue des études ergonomiques et reléguée à la sphère privée. Voulant analyser cette activité de conciliation associée inéluctablement au travail, nous avons étudié, avec la Fédération des travailleurs et travailleuses du Québec (FTQ), des téléphonistes mères de jeunes enfants et sujettes à un horaire variable et imprévisible (Prévost et coll., 1997). Trente téléphonistes parents de jeunes enfants ont rempli un journal de bord pendant 2 semaines, où elles ont rapporté leur horaire, leurs démarches d'arrangement de garde et leurs démarches de changement d'horaire. Ces informations ont été complétées par des entrevues 
téléphoniques, par deux entrevues collectives et par des discussions avec des cadres et des représentantes syndicales. Nous avons trouvé que les téléphonistes ont des horaires très variables. Par exemple, une téléphoniste peut commencer à $8 \mathrm{~h}$ le lundi, $16 \mathrm{~h}$ le mardi et $6 \mathrm{~h}$ le mercredi et les jours de congé hebdomadaire varient autant. Elle reçoit cet horaire trois jours avant son début, et est donc, contrainte à assurer la garde de son enfant dans de très brefs délais.

Face à cette contrainte, les téléphonistes déploient plusieurs stratégies pour maintenir leur disponibilité complète pour l'employeur en conciliant leur horaire de travail avec l'exigence de présence auprès de jeunes enfants. Elles font des démarches en vue de changement d'horaire (un total de 156 pendant la période pour les 30) avec un taux de succès d'un changement sur 5,3 démarches; elles «banquent » du temps; elles soignent leur relation avec leur superviseur; elles créent un réseau de garde qui comprend jusqu'à 8 personnes, elles-mêmes obligées d'avoir une grande souplesse dans les horaires (212 réarrangements de garde en tout); plusieurs décalent leur horaire avec celui de leur conjoint ou travaillent de soir. Énormément de temps, d'énergie et de créativité des téléphonistes ayant de jeunes enfants sont absorbés par la conciliation des horaires de travail avec la vie familiale.

Rien de tout cet effort n'est reconnu par l'employeur, qui montre une rigidité prononcée face aux difficultés vécus par les employées. On ne permet pas à l'employée d'appeler ou de recevoir des appels téléphoniques au travail, même en cas d'urgence. Le moindre retard est noté au dossier. Ce qui est très frappant, c'est que tous, superviseurs et téléphonistes, partagent le point de vue que c'est à l'employée de se débrouiller avec ses problèmes de garde. Alors que des besoins " personnels » communs aux hommes et aux femmes, comme la nécessité de prendre des pauses, de prendre un repas, de dormir ou de faire ses besoins, sont reconnus dans les milieux de travail et font l'objet de dispositions réglementaires, tel n'est pas le cas des besoins de protection des enfants et des aînés ressentis le plus souvent par des travailleuses (accès au téléphone, horaires qui ne varient pas trop, tolérance des retards inévitables, stabilité des congés hebdomadaires). Les difficultés liées à la conciliation paraissent comme des problèmes personnels, alors qu'elles découlent directement des conditions de travail.

\section{Travail émotionnel et emplois des femmes}

Une étude du travail des préposées à l'accueil d'un hôpital, menée avec la CSN, nous a permis d'examiner les exigences d'un travail émotionnel, défini par Hochschild (1983) comme celui qui

«produit l'état d'esprit voulu chez les autres».

La tâche des préposées est d'amener les bénéficiaires à l'hôpital aux moments prévus et de s'assurer qu'ils aient fait ce qu'on leur demande (être à jeun, par exemple). Les bénéficiaires doivent faire face à de longues attentes, de l'incertitude quant au diagnostic et quant au moment du traitement et parfois au fait de voir une intervention recédulée à la dernière minute. Certains ont peur tandis que d'autres ont hâte à l'intervention. Ils ont des degrés divers de facilité à parler au téléphone et de compréhension de la langue française. Nous avons analysé les communications entre préposées, les postures des membres supérieurs, et les contenus de certains échanges avec les bénéficiaires (Seifert et coll., 1996b). 

aurait une importance certaine pour la transformation du travail des femmes.

\section{Que fait l'ergonome de la variable « sexe »?}

51 Ainsi, dans plusieurs milieux de travail, le sexe représente des opérations spécifiques à effectuer, des contraintes additionnelles et des attentes de la part des collègues et des superviseurs quant aux modes opératoires. S'il n'y a pas d'analyse fine de toutes les composantes de l'activité, les scientifiques qui voient que les travailleuses et les travailleurs n'ont pas les mêmes maladies concluront à telle influence hormonale, telle faiblesse associée au sexe ou telle propension à se plaindre. Pour l'ergonome, il sera 
pertinent de tenir compte du sexe des opérateurs-trices à plusieurs moments pendant une intervention:

1. Au moment du travail de la demande. Au début d'une étude ergonomique, on essaie de recueillir des informations sur le besoin d'une intervention. Les hommes et les femmes peuvent avoir des intérêts différents, même à l'intérieur d'un métier. Ils peuvent avoir des problèmes de santé différents, en fonction d'opérations ou de modes opératoires caractéristiques. Comme les femmes sont habituellement exclues des postes où les dangers sont impressionnants et dramatiques, il sera facile de négliger leurs problèmes. Lors des entrevues avec les superviseurs, il sera important de dégager leurs attentes face aux travailleurs des deux sexes. Il sera peut-être opportun de rencontrer les travailleuses et les travailleurs séparément, afin de connaître les problèmes dont ils ne peuvent pas parler ensemble (fonctionnement sexuel, harcèlement sexuel, problèmes de menstruations, liés à la grossesse, à la famille, etc.).

2. Au moment des observations et des entretiens. C'est à ce moment qu'on peut relever les tâches assignées différemment selon le sexe et également les modes de répartition du travail à l'intérieur des équipes. On pourra voir le degré de prise en compte de responsabilités familiales et observer les opérations visant à concilier travail et famille. On peut identifier des modes opératoires spécifiques dont les déterminants se retrouvent dans les attentes différentes du public et de l'employeur face aux femmes et aux hommes. Il sera important de relever les compétences méconnues qui sont mises en œuvre pendant des opérations en apparence banales, aussi bien que les exigences physiques, mentales et émotionnelles peu visibles.

3. Au moment de la restitution. Il sera important dans certains cas de réunir séparément les travailleuses et les travailleurs en plus de les voir ensemble. D'abord parce que les travailleuses ont tendance à parler moins dans des groupes mixtes et aussi parce que des pistes de solutions qui paraissent très appropriées pour des personnes sans responsabilités familiales peuvent poser de sérieux problèmes pour celles qui ont la responsabilité d'enfants ou de vieux parents. Comme il est interdit de laisser la famille empiéter sur le travail (mais pas l'inverse), il sera critique de créer une atmosphère où les personnes ayant de lourdes responsabilités familiales se sentent en mesure de s'exprimer. En complément, il sera important de pouvoir discuter avec les travailleuses du fait que certaines solutions qui peuvent paraître intéressantes du point de vue des responsabilités familiales (horaire différentiel selon la constellation familiale, exclusion de certaines tâches pour les femmes) pourraient mettre en péril le droit des femmes au travail. Il sera aussi pertinent de confronter certains stéréotypes qui peuvent entraver la mise en marche des solutions. Dans le cas où il faut combattre des stéréotypes, il peut être pertinent d'impliquer des ergonomes des deux sexes dans la restitution des résultats. Ceci est particulièrement important afin que la discussion des questions reliées au sexe des travailleurs puisse devenir une partie ouverte et publique de la restitution.

4. Au moment de la formation. Si l'intervention donne lieu à un programme de formation, il sera pertinent de voir à ce que des travailleuses puissent en profiter. Puisqu'elles se sentent souvent exclues de la vie syndicale, des activités en santé au travail et des programmes de qualification, assurer leur participation exige souvent un effort spécifique. Il sera aussi important d'inclure leurs préoccupations particulières dans le programme de formation.

\section{Conclusion}

52 Au CINBIOSE, nous avons analysé le travail dans plusieurs secteurs d'emploi: la transformation des poissons, la couture, la confection de bottes, la transformation de la volaille, le tri des lettres et des colis, la boulangerie, le montage de moteurs, le nettoyage, 
le jardinage, l'accueil des patients-es dans un hôpital, les soins de santé, la caisse de supermarché, l'enseignement, la caisse de banque, les techniques de laboratoire et les soins. Nous avons appris que la prise en compte du sexe des opérateurs enrichit l'analyse du travail et que l'analyse ergonomique enrichit la compréhension de la division sexuelle $\mathrm{du}$ travail. Cette perspective n'est pas toujours bienvenue dans les milieux de travail où les hommes et les femmes peuvent avoir un vécu et des intérêts divergents. Les discussions n'ont pas toujours été agréables ni les transformations acceptées facilement. Mais nous pensons qu'il est important de mettre ces questions sur la place publique afin de transformer certains aspects pénibles du travail des femmes.

\section{BIBLIOGRAPHIE}

Armstrong, P., Armstrong, H. (1993). The Double Ghetto : Canadian Women and their Segregated Work. 3rd edition, Toronto- McClelland and Stewart.

Asselin, S., Gauthier, H., Lachapelle, J., Lavoie, Y., Duchesne, L., Jean, S., Laroche, D., Nobert, Y. (1994). Les hommes et les femmes: Une comparaison de leurs conditions de vie. Bureau de la statistique du Québec, Québec.

Boucher, M., Messing, K., Courville, J. (1994). Analyse du travail des jardinières et des jardiniers à la ville X. CINBIOSE, Montréal.

Boucher, M. (1995). Analyse de l'activité des jardiniers et des jardinières cols bleus. Mémoire de maîtrise, Département des sciences biologiques, UQAM.

Carpentier-Roy, M.-C. (1991). Corps et Âme. Liber, Montréal.

Chatigny, C., Seifert, A. M., Messing, K. (1995). Repetitive movements in a non-repetitive task : A case study. International Journal of Occupational Safety and Ergonomics, 1, 1, 42-51.

Cloutier, E., Duguay, P. (1996). Impact de l'avance en âge sur les scénarios d'accidents et les indicateurs de lésions dans le secteur de la santé et des services sociaux, tome I. IRSST, R-118, 185p.

Courville, J., Dumais, L., Vézina, N. (1994). Conditions de travail de femmes et d'hommes sur une chaîne de découpe de volaille et développement d'atteintes musculo-squelettiques. Travail et Santé, 10, 3, S17-S23.

Courville, J., Vézina, N., Messing, K. (1992). Analyse des facteurs ergonomiques pouvant entraîner l'exclusion des femmes du tri des colis postaux. Le travail humain, 55, 119-134.

Courville, J., Vézina, N., Messing, K. (1991). Comparison of the work activity of a two mechanics : A woman and a men. International Journal of Industrial Ergonomics, 7, 63-174.

David, H. (1986). Femmes et emploi : le défi de l'égalité. Presses de l'Université du Québec, Québec.

De Koninck, M. (1983). La santé des travailleuses. Ministère des affaires sociales, Québec.

Derriennic, F., Touranchet, A., Volkoff, S. (1996). Age, travail, santé : Études sur les salariés âgés de 37 à 52 ans. Les éditions INSERM, Paris. 
Dessors, D., Teiger, C., Laville, A., Gadbois, C. (1978). Conditions de travail des opératrices des renseignements téléphoniques et conséquences sur leur vie personnelle et sociale. Arch. mal. prof., 40, 3-4, 469-500.

Doniol-Shaw, G. (1983). L'ergonomie du travail ménager. Groupe d'étude de la division sociale et sexuelle du travail. Conseil national de la recherche scientifique, Paris.

Dumais, L., Courville, J. (1995). Aspects physiques de la division sexuelle des tâches : Quand la qualification professionnelle et l'organisation du travail viennent en aide aux femmes cols bleus. Revue canadienne de sociologie et d'anthropologie, 32, 4, 385-414.

Dumais, L., Messing, K., Seifert, A. M., Courville, J.,Vézina, N. (1993). Make me a cake as fast as you can : Determinants of inertia and change in the sexual division of labour of an industrial bakery. Work, Employment and Society, 7, 3, 363-382.

Falzon, P. (1997). L'ergonomie des services. Performances humaines et techniques, 89, 6-7.

Falzon, P., Lapeyrière, S. (1998). L'usager et l'opérateur : Ergonomie et relations de service. Le travail humain, 61, 1, 69-90.

Gaucher, D. (1983). Le maternage mal salarié : La division sexuelle du travail en milieu hospitalier. Les Presses de l'Université de Montréal, Montréal.

Gonik, V., Cardia-Vonèche, L., Bastard, B., von Allmen, M. (1998). Construire l'égalité : femmes et hommes dans l'entreprise. Georg Éditeur, Chêne-Bourg, Suisse.

Guérin, F., Laville, A., Daniellou, F., Duraffourg, J., Kerguelen, A. (1991). Comprendre le travail pour le transformer. ANACT, Montrouge.

Guyon, L. (1996). Derrière les apparences : santé et conditions de vie des femmes. Ministère de la santé et des services sociaux, Québec.

Hochschild, A.R. (1983). The managed heart : Commercialization of human feeling. University of California Press, Berkeley.

Kergoat, D. (1983). Les ouvrières. Sycomore, Paris.

Kilbom, Å., Messing, K., Thorbjornsson, C. (1998). Women's Health at Work. National Institute for Working Life, Solna, Suède.

Kilbom, Å., Torgén, M. (1996). Do physical loads at work interact with aging ? Communication le 18 septembre 1996 au 25e Congrès international sur la médecine du travail, tenu à Stockholm, Actes, tome 1, p. 17.

Laberge, M., Vézina, N. (1998). Un banc assis-debout pour les caissières. Travail et santé, 14, 2, $42-48$.

Laurin, G. (1991). Féminisation de la main d'œuvre. Impact sur la santé et la sécurité du travail. CSST, Montréal.

Le Bourdais, C., Hamel, P.J., Bernard, P. (1987). Le travail et l'ouvrage. Charge et partage des tâches domestiques chez les couples québécois. Sociologie et Sociétés, 19, 1, 37-56.

Lippel, K., Bernstein, S., Bergeron, M.C. (1996). Le retrait préventif de la travilleuse enceinte ou qui allaite : réflexions sur le droit et la médecine. Les Éditions Yvon Blais - Cowansville, Québec.

Lippel, K., Messing, K., Stock, S., Vézina, N. (sous presse). La preuve de la causalité et l'indemnisation des lésions attribuables au travail répétitif: rencontre des sciences de la santé et du droit. Windsor Yearbook of Access to Justice, 17. 
Lortie, M. (1987). Analyse comparative des accidents déclarés par des préposés hommes et femmes d'un hôpital gériatrique. Journal of Occupational Accidents, 9, 59-81.

Malenfant, R. (1996). Travail et grossesse : Peut-on laisser la maternité à la porte de l'entreprise. Liber, Montréal.

Messing, K. (1996). Le genre des « opérateurs » : Est-ce un paramètre pertinent pour l'analyse du travail ? Les cahiers du MAGE, 4, 96, 45-60.

Messing, K. (1998). One-eyed Science : Occupational Health and Women Workers. Temple University Press, Philadelphia.

Messing, K., Boutin, S. (1997). La reconnaissance des conditions difficiles dans les emplois des femmes et les instances gouvernementales en santé et en sécurité du travail. Relations industrielles , 52, 2, 333-362.

Messing, K., Chatigny, C., Courville, J. (1998b). "Light" and "heavy" work in the housekeeping service of a hospital. Applied Ergonomics, 29, 6, 51-459.

Messing, K., Courville, J., Boucher, M., Dumais, L., Seifert, A.M. (1994b). Can safety risks of bluecollar jobs be compared by gender? Safety Science, 18, 95-112.

Messing, K., dir. (1999). Comprendre le travail des femmes pour le transformer. Bureau technique syndical de la Communauté économique européenne, Bruxelles.

Messing, K., Dumais, L., Courville, J., Seifert, A.M., Boucher, M. (1994a). Evaluation of exposure data from men and women with the same job title. J. Occup. Medicine, 36, 8, 913-917.

Messing, K., Elabidi, D. (1998). Collaboration entre les travailleurs lors d'efforts physiques. Objectif Prévention, 21, 5, 24-26.

Messing, K., Haëntjens, C., Doniol-Shaw, G. (1993). L'invisible nécessaire :l'activité de nettoyage des toilettes sur les trains de voyageurs en gare. Le travail humain, 55, 353-370.

Messing, K., Seifert, A.M., Escalona, E. (1997). The 120-second minute : Using analysis of work activity to prevent psychological distress among elementary school teachers. Journal of Occupational Health Psychology, 2, 1, 45-62.

Messing, K., Tissot, F., Saurel-Cubizolles, M.J., Kaminski, M., Bourgine, M. (1998a). Sex as a variable can be a surrogate for some working conditions : Factors associated with sickness absence. Journal of Occupational and Environmental Medicine, 40, 250-260.

Paul, J.M. (1993). Pregnancy and the Standing Working Posture : An Ergonomic Approach. Coronel Laboratory, University of Amsterdam, Amsterdam, Pays-Bas.

Prévost, J., Messing, K. (1997). Quel horaire, What schedule ? L'horaire de travail irrégulier des téléphonistes. Dans, Stratégies de résistance et travail des femmes, Soares, Angelo (dir.), pp. 251-270, Harmattan, Montréal et Paris.

Saurel-Cubizolles, M.J., Blondel, B. (1996). La santé des femmes. Flammarion, Paris.

Saurel-Cubizolles, M.J., Bourgine, M., Touranchet, A., Kaminski, M. (1991). Enquête dans les abattoirs et les conserveries des régions Bretagne et Pays-de-Loire. Conditions de travail et santé des salariés. Villejuif, France, INSERM, Unité 149, 42p.

Saurel-Cubizolles, M.J., Messing, K., Lert, F. (1996). Activité professionnelle et santé des femmes. Dans, Saurel-Cubizolles, M.J. et Blondel, B. La santé des femmes, Flammarion - Paris.

Seifert, A.M., Messing, K., Dumais, L. (1996a). Les caissières dans l'œil du cyclone : analyse de l'activité de travail des caissières de banque. CINBIOSE, Montréal, 86p. 
Seifert, A.M., Messing, K., Dumais, L. (1997). Star wars and strategic defense initiatives : Work activity and health symptoms of unionized bank tellers during work reorganization. International Journal of Health Services, 27, 3, 455-477.

Seifert, A.M., Messing, K., Elabidi, D. (1996b). Analyse du travail des préposées à l'accueil de l'Hôpital X. CINBIOSE, Montréal, 67p.

Soares, A. (1995). Nouvelles technologies : nouvelles qualifications ? Le cas des caissières de supermarché. Recherches féministes, 9, 1, 37-56.

Teiger, C., Plaisantin, M.-C. (1984). Les contraintes du travail dans les travaux répétitifs de masse et leurs conséquences sur les travailleuses. Dans, Les effets des conditions de travail sur la santé des travailleuses, dir. Bouchard, Jeanne-Anne, 33-68, Confédération des syndicats nationaux, Montréal.

Teiger, C., Bernier, C. (1992). Ergonomic analysis of work activity of data entry clerks in the computerized service sector can reveal unrecognized skills. Women and Health, 18, 3, 67-78.

Tissot, F., Messing, K., Vandelac, L., Garon, S., Prévost, J., Méthot, A.-L., Pinard, R. (1997). Concilier les responsabilités professionnelles, familiales, personnelles et sociales, ce n'est pas toujours la santé. Fédération des travailleurs et travailleuses du Québec, CINBIOSE, Montréal. 52p.

Vandelac, L. (1985). Du travail et de l'amour. Éditions St-Martin, Montréal.

Vézina, N., Chatigny, C., Messing, K. (1994). Un poste de manutention : symptômes et conditions de travail chez les caissières de deux supermarchés. Maladies chroniques au Canada, 15, 1, 19-24.

Vézina, N., Courville, J., Geoffrion, L. (1995). Problèmes musculo-squelettiques, caractéristiques des postes de travailleurs et des postes de travailleuses sur une chaîne de découpe de dinde. Dans, Invisible : Issues in Women's Occupational Health and Safety/Invisible : La santé des travailleuses. Messing, K., Neis, B. and Dumais, L. (dir.) Gynergy Books - Charlottetown, 29-61.

Wisner, A., Laville, A., Richard, E. (1967).Conditions de travail des femmes O. S. dans la construction électronique. Laboratoire de Physiologie du Travail et Ergonomie du CNAM, rapport 2.

\section{RÉSUMÉS}

Souvent, les publications et communications à partir d'études ergonomiques sont muettes sur le sexe des «opérateurs". Il n'est pas évident à première vue que le sexe soit une variable pertinente pour l'analyse du travail. Les postes étant toujours occupés par des personnes ayant des caractéristiques diverses, la tâche de l'ergonome consiste à assurer que le travail soit faisable par tous et par toutes. Je suggère cependant qu'il faut tenir compte du sexe dans les études ergonomiques. Les postes, les tâches, voire même les modes opératoires peuvent différer selon le sexe. Je conclus que la considération du sexe des "opérateurs" fournit une façon d'enrichir l'analyse ergonomique. Je suggère qu'il est pertinent pour l'analyse de la demande, la poursuite des observations, la restitution et la formation. Les exemples utilisés pour démontrer ces thèses proviennent des recherches effectuées au CINBIOSE depuis 10 ans, le plus souvent dans le cadre d'un programme de recherche mené en collaboration avec les trois principales centrales syndicales du Québec.

Often, publications and communications based on ergonomic studies do not mention the gender of the workers studied. At first glance, it is not obvious that gender is relevant for the analysis of work activity. Since the worker population has a variety of features, the ergonomist's task is to adapt worksites for all occupants, regardless of gender. Nevertheless, I propose that gender must 
be taken into account in ergonomic studies. Job and task assignments may differ according to sex, and gender-related characteristics may also be relevant if we consider the means different workers use to accomplish their assigned operations. I conclude that considering the gender of workers is a good way of enriching ergonomic analysis. I suggest that gender is a relevant consideration at various stages in the analysis : when examining the need for intervention, when observing, when discussing the report and when preparing training sessions. The examples used to demonstrate these points are taken from studies done by the CINBIOSE laboratory over the past ten years, usually in collaboration with the three major Québec trade unions.

Muchas veces, las publicaciones y comunicaciones a partir de estudios ergonómicos no hablan del sexo de los «operadores». A primera vista, no es evidente que el sexo sea una variable pertinente para el análisis del trabajo. Ya que los empleos siempre están ocupados por personas con características diferentes, la tárea del ergonomista consiste en asegurarse de que el trabajo se pueda hacer por todas y todos. Sin embargo, sugiero que hace falta tomar en cuenta el sexo en los estudios ergonómicos. Los empleos, las táreas, incluso los modos operativos pueden diferir según el sexo. Concluyo que la consideración del sexo de los «operadores » proporciona una manera de enriquecer el análisis ergonómico. Sugiero que esta consideración es pertinente para el análisis de la demanda, la prosecución de las observaciones, la restitución y la formación. Los ejemplos utilizados para demostrar estas tesis proceden de investigaciones efectuadas en el CINBIOSE desde hace 10 años, la mayor parte del tiempo en el marco de un programa de investigación llevado en colaboración con las primeras tres centrales sindicales del Quebec.

\section{INDEX}

Mots-clés : ergonomie, sexe, soignantes, enseignement, conciliation travail-famille

Palabras claves : ergonomía, sexo, cuidadoras, enseñanza, conciliación entre trabajo y familia

Keywords : ergonomics, gender, nursing, education, work and family

\section{AUTEUR}

\section{KAREN MESSING}

Centre pour l'étude des interactions biologiques entre la santé et l'environnement (CINBIOSE), UQAM, C.P. 8888, Succursale Centre-Ville, Montréal (Québec), Canada H3C 3P8, messing.karen@uqam.ca 